\title{
Morfologia externa dos estágios imaturos de heliconíneos neotropicais: VII. Dryadula phaetusa (Linnaeus) (Lepidoptera, Nymphalidae, Heliconiinae)
}

\author{
Denis S. da Silva ${ }^{1}$, Lucas A. Kaminski²; Rafael Dell’Erba ${ }^{3} \&$ Gilson R. P. Moreira ${ }^{1}$
}

\begin{abstract}
'Departamento de Zoologia, Instituto de Biociências, Universidade Federal do Rio Grande do Sul, Avenida Bento Gonçalves, 9500, $91.501-970$ Porto Alegre-RS, Brasil. denisdasilva@pop.com.br e gilson.moreira@ufrgs.br

${ }^{2}$ PPG-Ecologia, Instituto de Biologia, Universidade Estadual de Campinas, Caixa Postal 6109, 13.083-970 Campinas-SP, Brasil. lucaskaminski@yahoo.com.br

${ }^{3}$ Departamento de Ciências Biológicas e da Saúde, IUniAraguaia, Universidade Federal do Mato Grosso, BR 100, km 3.5, 78.698-000 Pontal do Araguaia-MG, Brasil. rafaeldellerba@yahoo.com.br
\end{abstract}

\begin{abstract}
External morphology of the immature stages of Neotropical heliconians: VII. Dryadula phaetusa (Linnaeus) (Lepidoptera, Nymphalidae, Heliconiinae). The external features of egg, larva and pupa of Dryadula phaetusa (Linnaeus, 1758) are described and illustrated, based upon light and scanning electron microscopy.
\end{abstract}

KEYWORDS. Egg; larva; Passiflora misera; pupa; Orange banded heliconian.

RESUMO. Morfologia externa dos estágios imaturos de heliconíneos neotropicais: VII. Dryadula phaetusa (Linnaeus) (Lepidoptera, Nymphalidae, Heliconiinae). As estruturas externas do ovo, larva e pupa de Dryadula phaetusa (Linnaeus, 1758) são descritas e ilustradas, baseadas em microscopia óptica e eletrônica de varredura.

PALAVRAS-CHAVE. Borboleta do maracujá; larva; ovo; Passiflora misera; pupa.

Dryadula phaetusa (Linnaeus, 1758) é monotípica (Lamas 2004), com ampla distribuição na região Neotropical, desde o México até o sul do Brasil e Uruguai (Emsley 1963; DeVries 1987). Os adultos são encontrados em ambientes abertos, como campos e brejos, e eventualmente podem formar dormitórios noturnos em fileiras, por baixo de folhas de gramíneas (Brown 1992). Apresentam asas alaranjadas com faixas pretas, havendo dimorfismo sexual, sendo as fêmeas mais pálidas, até amareladas, e os machos com coloração intensa e presença de androcônias (DeVries 1987; Brown 1992). Pertence ao anel mimético mülleriano laranja, do qual também fazem parte outros heliconíneos (Araújo \& Valente 1981).

Várias espécies de Passifloraceae foram listadas como planta hospedeira de D. phaetusa para o Brasil (Brown \& Mielke 1972; Brown 1981; Brown 1992), mas ocorre preponderantemente em espécies de Passiflora Linnaeus do subgênero Decaloba (De Candolle) Reichenbach (= Passiflora Linnaeus subgênero Plectostemma Masters) (Benson et al. 1975). No Rio Grande do Sul, foi registrada apenas em Passiflora misera Humbold, Bompland \& Kunth, sendo os ovos depositados individualmente, sobre diversas partes da planta (Dell'Erba et al. 2005). As larvas constroem abrigos do tipo poleiro nos ínstares iniciais (Penz 1999), um comportamento relacionado à defesa contra formigas (Benson et al. 1975; Freitas \& Oliveira 1996).

O conhecimento da morfologia dos estágios imaturos, aliado com outros caracteres, têm mostrado grande importância sistemática e filogenética, proporcionando um melhor entendimento do relacionamento entre os taxa em Lepidoptera (e.g. Kitching 1985; Miller 1991; Penz 1999; Motta 2003; Freitas \& Brown 2004). Aspectos bionômicos e da morfologia dos estágios imaturos de $D$. phaetusa foram abordados nos trabalhos de Beebe et al. (1960), Fleming (1960) e Brown (1981). Entretanto, ainda é exíguo tal conhecimento, sendo a morfologia ultraestrutural externa até o presente momento desconhecida. Uma série de estudos vem sendo desenvolvida a respeito dos estágios imaturos de heliconíneos neotropicais (Antunes et al. 2002; Kaminski et al. 2002; Tavares et al. 2002; Paim et al. 2004; Silva et al. 2006; Kaminski et al. 2008); como parte destes, o presente trabalho objetiva descrever e ilustrar a morfologia externa dos estágios de ovo, larva e pupa de D. phaetusa, bem como a quetotaxia larval e a identificação morfométrica dos respectivos ínstares.

\section{MATERIALE MÉTODOS}

Os espécimes analisados foram obtidos a partir de adultos coletados na Estação Agronômica da UFRGS, Eldorado do

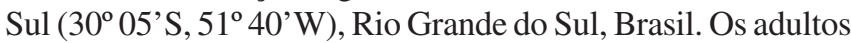
foram mantidos em insetário para a obtenção de ovos, que foram fixados para posterior análise ou acondicionados em placas de Petri, sobre papel filtro umedecido, até a eclosão. As larvas foram mantidas sobre ramos de $P$. misera e as cápsulas cefálicas, coletadas e separadas por ínstar. Os imaturos 
permaneceram em condições de laboratório $\left(25 \pm 1^{\circ} \mathrm{C}\right)$ durante todo o seu desenvolvimento. O material foi fixado em solução de Dietrich, conservado em etanol 70\%, observado em estereomicroscópio ou preparado para a microscopia eletrônica de varredura. Material testemunho foi depositado sob o número 103, na Coleção do Laboratório de Morfologia e Comportamento de Insetos (LMCI), Departamento de Zoologia, UFRGS, Porto Alegre, RS, Brasil.

A morfologia geral foi observada em uma lupa Spencer ${ }^{\circledR} \mathrm{e}$ ilustrada com o auxílio de uma grade micrométrica. As análises foram baseadas no material fixado ou incluso em gelatina glicerinada. As cápsulas cefálicas foram reidratadas, clarificadas em solução de hidróxido de potássio $(\mathrm{KOH})$ a $10 \%$, e montadas sobre lâmina/lamínula usando-se gelatina glicerinada como meio de inclusão. O padrão de coloração da larva de quinto ínstar e da pupa foram representados em aquarela, baseado na observação de espécimes vivos e/ou fotografias destes. Foi medida a maior largura da cápsula cefálica, em vista frontal. Os valores relativos foram ajustados pelo método dos mínimos quadrados à função: $\mathrm{y}=\mathrm{ae}^{\mathrm{bx}}$ (Snedecor \& Cochran 1980).

Para a morfologia ultraestrutural externa as amostras foram preparadas no Centro de Microscopia Eletrônica da UFRGS (CME) seguindo o protocolo: 1) desidratação em ponto crítico, 2) montagem sobre suporte metálico e 3 ) metalização com ouropaládio. As micrografias foram realizadas num microscópio eletrônico de varredura JEOL ${ }^{\circledR}$ JSM5800 no CME, com exceção da larva de primeiro ínstar que foi fotografada no Centro de Microscopia Eletrônica e Microanálise da ULBRA em um aparelho PHILIPS ${ }^{\circledR}$ XL20. As análises morfológicas foram baseadas na comparação de, no mínimo, cinco exemplares e, para a identificação morfométrica, em 20 larvas por ínstar.

$\mathrm{Na}$ descrição do ovo, utilizou-se a terminologia adotada por Dell'Erba et al. (2005). No estágio larval as áreas do corpo seguiram o proposto por Peterson (1962) e para a quetotaxia primária e ganchos dos larvópodos, Stehr (1987). Em relação às cerdas do paraprocto e da seção cilíndrica do larvópodo do décimo segmento abdominal, adotou-se Kitching (1984). Para os escolos larvais baseou-se em Beebe et al. (1960). Para a pupa, seguiu-se o proposto por Mosher (1916).

\section{RESULTADOS EDISCUSSÃO}

Ovo. Coloração amarela no início do desenvolvimento, tornando-se alaranjada com manchas marrons próximo à eclosão (Beebe et al. 1960; Brown 1981; Dell'Erba et al. 2005). Possui formato subcilíndrico, base plana e o ápice levemente afilado (Fig. 1). As dimensões, diâmetro e altura (média \pm erro padrão, $\mathrm{n}=10$ ) foram $1,121+0,015 \mathrm{~mm}$ e 1,606 + 0,015 mm, respectivamente. Estes valores são similares aos obtidos por Beebe et al. (1960) e Brown (1981).

O cório (Fig. 1) tem carenas verticais ( Vr) e horizontais (Hr), com larguras semelhantes e superfície lisa. O número de $\mathrm{Vr}$ varia de 17 a 19 e, de $\mathrm{Hr}$, de 15 a $16(\mathrm{n}=10)$. Tais valores são semelhantes aos encontrados por Beebe et al. (1960) e menores que os obtidos por Brown (1981) para Hr. As carenas delimitam células de superfícies lisas e ângulos internos arredondados, similares àquelas observadas em espécies de Agraulis Boisduval \& Le Conte, Dione Hübner, Dryas Hübner e Philaethria Billberg (Dell'Erba et al. 2005). As células inferiores (Lc) (Fig. 2) são retangulares. As superiores (Uc) (Fig. 3) podem ser retangulares, tetragonais irregulares ou pentagonais. As aerópilas (Ac) (Fig. 4) situam-se nas intersecções das carenas, são circulares e sem elevações no peritrema (Dell'Erba et al. 2005). Semelhante a outros Heliconiinae, a região micropilar situa-se no pólo anterior do ovo (Fig. 5). Possui formato
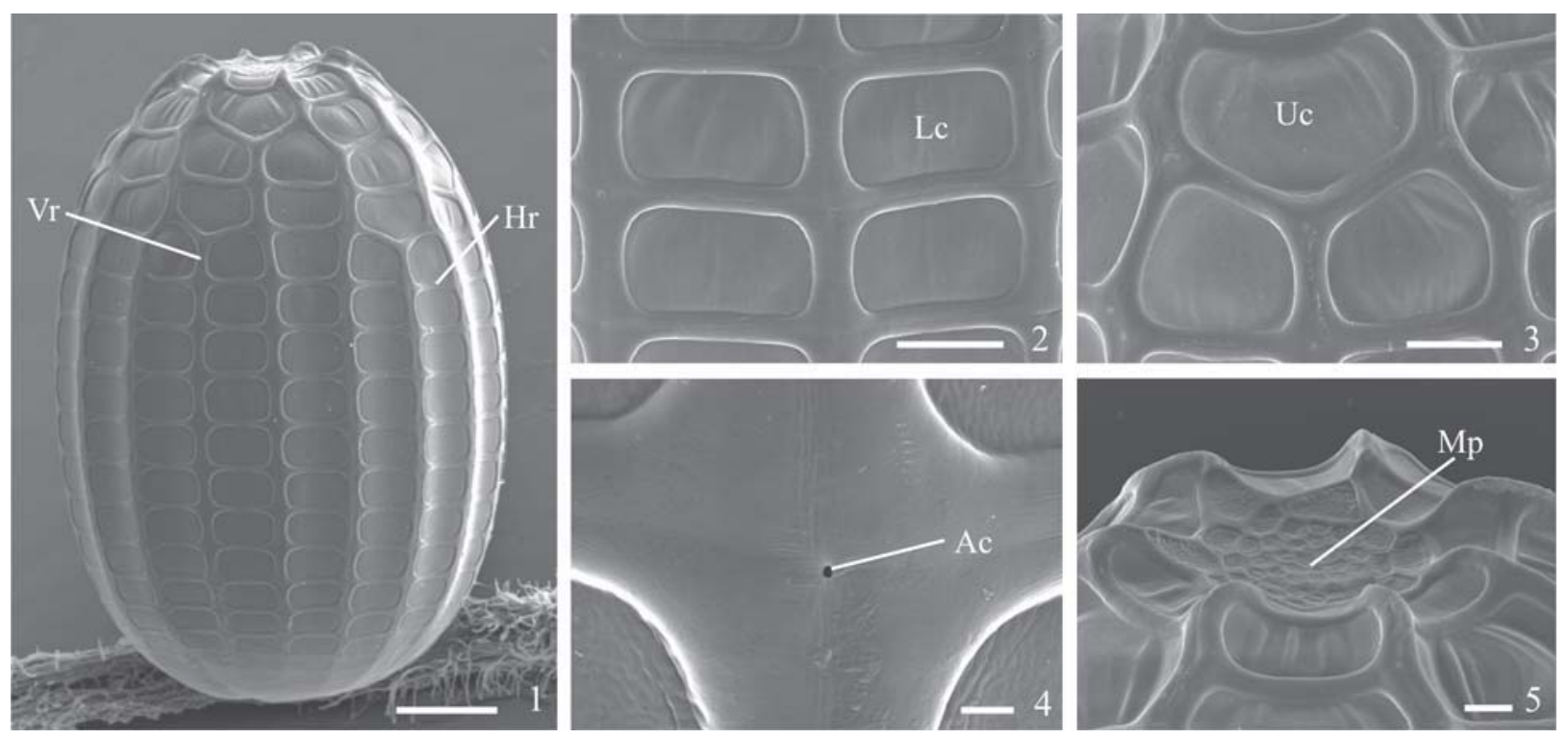

Figs. 1-5. Ovo de Dryadula phaetusa (Linnaeus, 1758). 1, vista lateral; 2, células inferiores; 3, células superiores; 4, aerópila; 5, região micropilar. Ac, aerópila; Hr, carena horizontal; Lc, célula inferior; Mp, micrópilas; Uc, célula superior; Vr, carena vertical. Barras $=250,100,100$, 10 e 50 $\mu \mathrm{m}$, respectivamente. Modificado de Dell'Erba et al. 2005. 
Tabela I. Média aritmética e erro padrão, intervalo de variação e razão de crescimento da largura da cápsula cefálica dentre os ínstares larvais de Dryadula phaetusa (Linnaeus, 1758), criados sobre Passiflora misera Humbold, Bompland \& Kunth, $\mathrm{n}=20$ /instar.

\begin{tabular}{cccc}
\hline & \multicolumn{3}{c}{ Largura da cápsula cefálica $(\mathrm{mm})$} \\
\cline { 2 - 4 } Instar & Média $\pm \underline{\mathrm{EP}}$ & $\begin{array}{c}\text { Intervalo de } \\
\text { Variação }\end{array}$ & $\begin{array}{c}\text { Razão de } \\
\text { Crescimento }\end{array}$ \\
\hline I & $0,701 \pm 0,008$ & $0,640-0,760$ & --- \\
II & $1,052 \pm 0,007$ & $1,000-1,100$ & 1,50 \\
III & $1,593 \pm 0,009$ & $1,500-1,640$ & 1,51 \\
IV & $2,345 \pm 0,016$ & $2,185-2,470$ & 1,47 \\
V & $3,679 \pm 0,042$ & $3,286-3,922$ & 1,57 \\
\hline
\end{tabular}

côncavo com nítidas carenas verticais elevadas. A região micropilar é formada pelo ânulo e a roseta, e no centro desta, estão localizadas as micrópilas (Mp) (Downey \& Allyn 1981). As células desta região apresentam formato que varia do tetragonal ao hexagonal com suaves rugosidades na superfície.

Larvas. No primeiro ínstar, a larva apresenta coloração marrom-clara com manchas brancas e faixas marrom-escuras no tórax e abdômen. A cápsula cefálica é marrom-translúcida. As placas, pináculas, calazas e cerdas maiores variam de marrom-escuras a pretas, já as cerdas menores são hialinas.

Fronte e clípeo fusionados, formando um frontoclípeo com

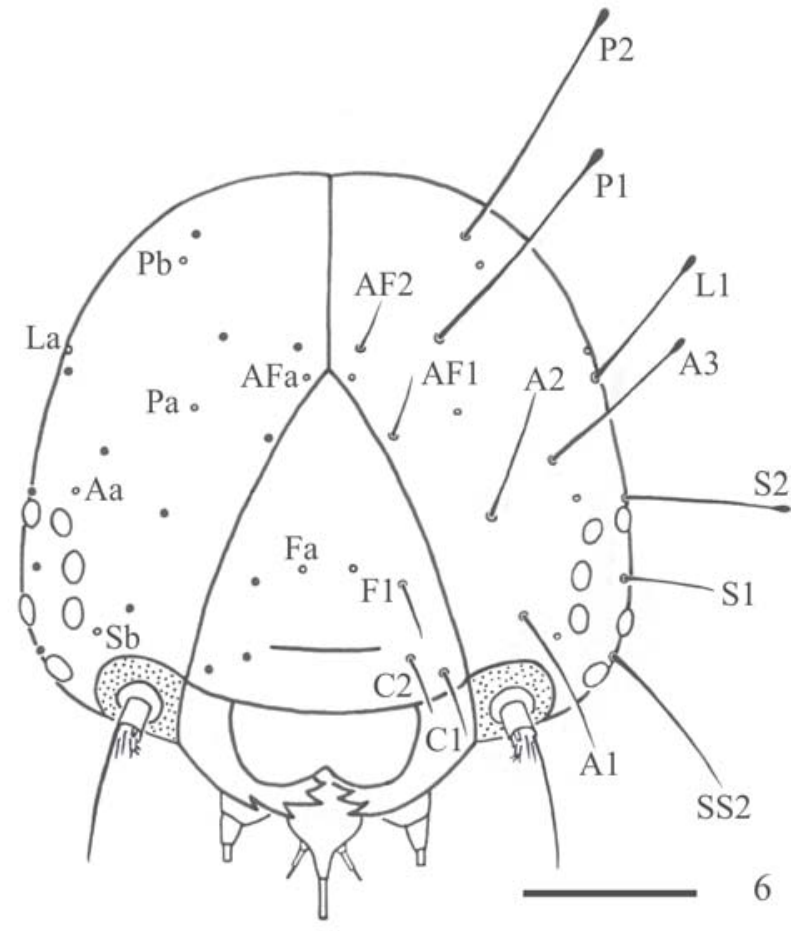

Fig. 6. Vista frontal da cápsula cefálica da larva de primeiro ínstar de Dryadula phaetusa (Linnaeus, 1758). A, grupo anterior; AF, grupo adfrontal; $\mathrm{C}$, cerda clipeal; F, grupo frontal; L, grupo lateral; $\mathrm{P}$, grupo póstero-dorsal; $\mathrm{S}$, grupo estematal; SS, grupo subestematal. Barra $=151 \mu \mathrm{m}$.
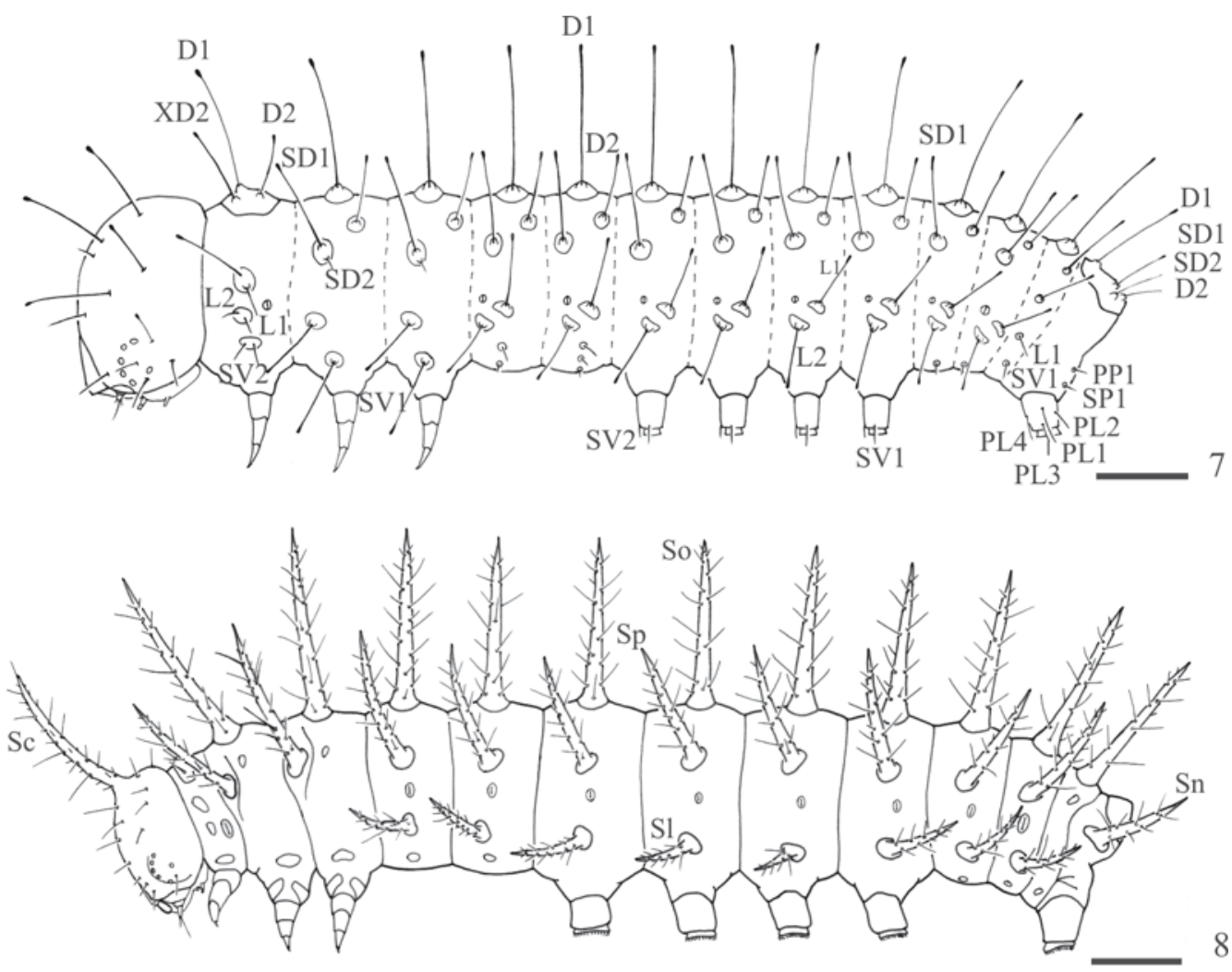

Figs. 7-8. Representação esquemática das larvas de Dryadula phaetusa (Linnaeus, 1758) em vista lateral. 7, primeiro ínstar; 8, quinto ínstar. D, cerda dorsal; L, cerda lateral; PL, cerda da seção cilíndrica do larvópodo do décimo segmento abdominal; PP, cerda paraproctal; Sc, escolo cefálico; SD, cerda subdorsal; Sl, escolo subespiracular; Sn, escolo anal; So, escolo dorsal; SP, cerda subparaproctal; Sp, escolo supraespiracular; SV, cerda subventral; XD, cerda protorácica. Barras $=0,25$ e $2,5 \mathrm{~mm}$, respectivamente. 

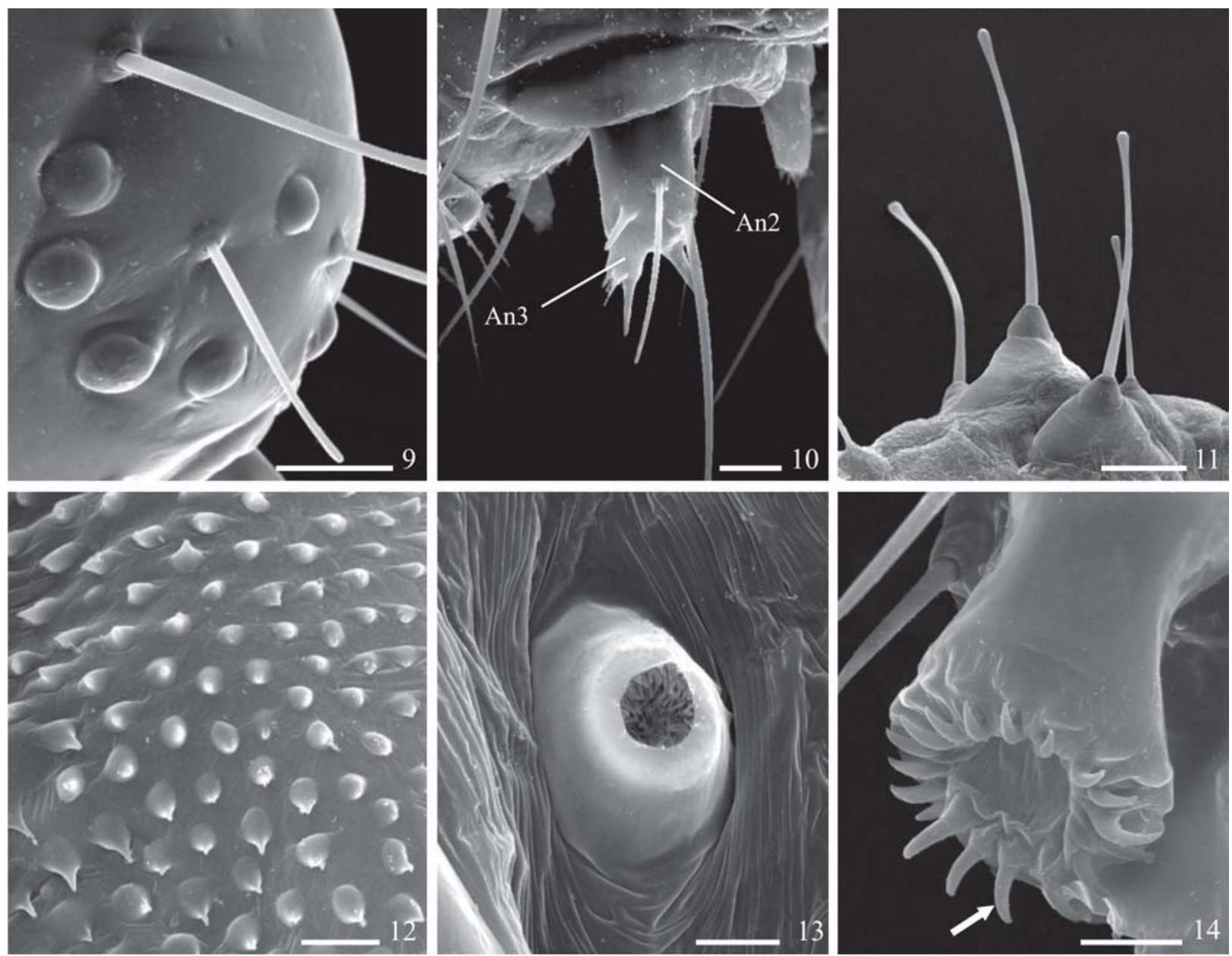

Figs. 9-14. Larva de primeiro ínstar de Dryadula phaetusa (Linnaeus, 1758). 9, região estematal em vista látero-dorsal; 10, antena; 11, cerdas sobre calazas; 12, microtríquias; 13, espiráculo; 14, planta do larvópodo com ganchos (seta). An, artículo antenal. Barras = 50, 25, 100, 10, 10, $25 \mu \mathrm{m}$, respectivamente.

sutura frontoclipeal pouco evidente. O anteclípeo não é aparente em vista frontal, semelhante a Heliconius erato phyllis (Fabricius, 1775) e Dryas iulia alcionea (Cramer, 1779) (Kaminski et al. 2002; Paim et al. 2004), mas contrastando com outros heliconíneos já estudados que apresentam o anteclípeo bem aparente (Antunes et al. 2002; Tavares et al. 2002; Silva et al. 2006; Kaminski et al. 2008). Labro bilobado. Estematas (Fig. 9), com tamanhos similares e dispostos em semicírculo. Um tênue facetamento em forma de "y" nas lentes e no sulco que margeia anteriormente cada estemata é visível desde o primeiro ínstar, diferente de outros Heliconiini que apresentam tais características evidentes a partir do segundo ínstar. Antena (Fig. 10) formada por três artículos e inserida em uma área membranosa eversível, a antacória.

A quetotaxia primária (Figs. 6-7) é semelhante à descrita para outros heliconíneos (Fleming 1960; Antunes et al. 2002; Kaminski et al. 2002; Tavares et al. 2002; Paim et al. 2004; Silva et al. 2006). As cerdas são lisas, sobre calazas e com a porção apical dilatada (Fig. 11), com exceção das cerdas menores, que possuem o ápice afilado. Cerdas primárias com o ápice dilatado têm sido descritas para diversos Heliconiini
(Kaminski et al. 2002; Paim et al. 2004; Silva et al. 2006; Kaminski et al. 2008). Porém, a morfologia do ápice dessas cerdas pode variar em outras espécies, sendo afilada em Eueides isabella dianasa (Hübner, [1806]) (Antunes et al. 2002), e partida em Dione juno juno (Cramer, 1779) (Tavares et al. 2002).

Na cápsula cefálica (Fig. 6), os pares de cerdas, microcerdas e poros, estão reunidos em dez grupos. Grupo anterior (A): cerdas A1, A2 e A3; poro Aa, próximo ao segundo estemata. Grupo adfrontal (AF): cerdas AF1 e AF2, junto à porção superior do frontoclípeo; poro AFa posicionado medianamente entre as duas cerdas adfrontais. Grupo clipeal (C): cerdas C1 e C2. Grupo céfalo-dorsal (CD sensu Duarte et al. 2005): não visível em vista frontal, mas representado pelas microcerdas $\mathrm{CD} 1, \mathrm{CD} 2$ e CD3, localizadas posteriormente à $\mathrm{P} 2$; poro $\mathrm{CDa}$ entre CD1 e CD2. Grupo frontal (F): cerda F1; poro Fa, localizado entre as F1. Grupo lateral (L): cerda L1; poro La, localizado posteriormente à L1. Grupo microgenal (MG): com a microcerda MG1; e poro MGa. Grupo póstero-dorsal (P): cerdas P1 e P2 longas; poro $\mathrm{Pa}$, anterior a $\mathrm{P} 1$, e poro $\mathrm{Pb}$, situado entre $\mathrm{P} 1$ e $\mathrm{P} 2$. Grupo estematal (S): cerdas S1, S2 e S3; poros Sa e Sb. Grupo subestematal (SS): cerdas SS1, SS2 e SS3; poros SSa e SSb. 

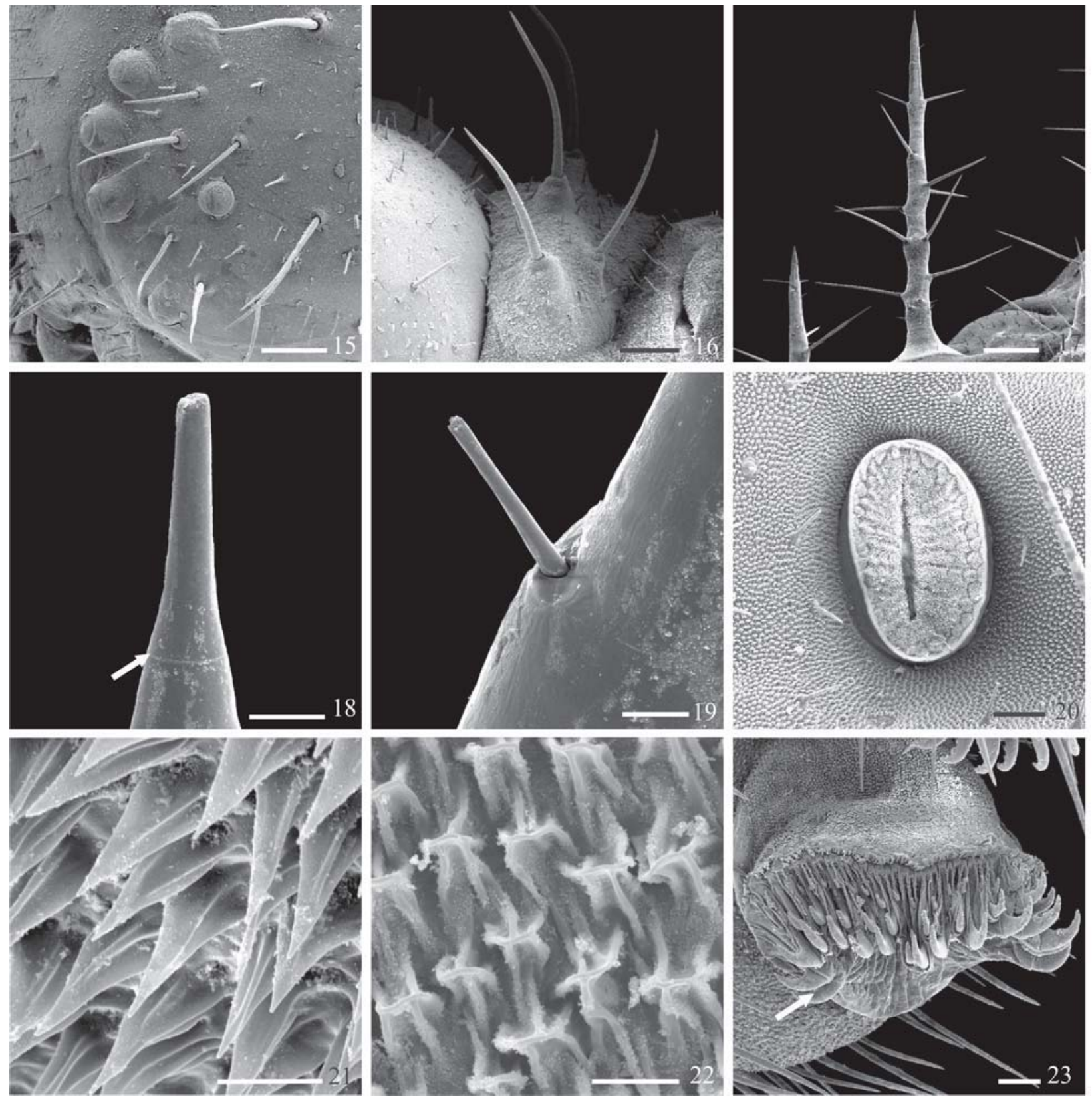

Figs. 15-23. Larva de quinto ínstar de Dryadula phaetusa (Linnaeus, 1758). 15, região estematal em vista lateral; 16, placa protorácica em vista lateral; 17, escolo dorsal; 18, porção terminal de um escolo, note a suave delimitação da área de inserção da cerda (seta); 19, detalhe de uma cerda do escolo; 20, espiráculo; 21, microtríquias próximas ao larvópodo; 22, microtríquias próximas ao espiráculo; 23, planta do larvópodo com ganchos (seta). Barras $=200,200,500,50,20,100,10,10$ e $100 \mu \mathrm{m}$, respectivamente.

No labro, mandíbula e maxila também estão presentes cerdas menores que não foram nomeadas (Stehr 1987).

Quetotaxia do tórax e abdômen com os seguintes grupos de cerdas: protorácica (XD), dorsal (D), subdorsal (SD), lateral (L), ventral (V), subventral (SV), paraproctal (PP), subparaproctal (SP), tarsal (Ts), e da seção cilíndrica do larvópodo do décimo segmento abdominal (PL). O grupo ventral (V) é formado por cerdas curtas e finas (Fleming 1960), presentes em alguns segmentos do corpo da larva. Poros e microcerdas também estão nele presentes, mas não foram descritos na quetotaxia (Kitching 1984).
Protórax: placa protorácica com cerdas do grupo dorsal, D1 e D2, sendo a primeira, a mais longa da placa; grupo protorácico com a cerda XD2, e os poros XDc (Fleming 1960) e XDb (Antunes et al. 2002; Paim et al. 2004). Cerdas subdorsais SD1 e SD2, na mesma pinácula, em posição ânterodorsal ao espiráculo, com SD1 mais longa. Cerdas laterais L1 e L2 inseridas em uma pinácula anterior ao espiráculo. Cerdas subventrais SV2 e SV1 em uma mesma pinácula, com SV2 anterior à SV1, e ambas de tamanho semelhante às cerdas laterais. Nas pernas ocorrem cerdas tibiais e as tarsais Ts1, Ts2, Ts3 e Ts4 (sensu Miller 1991), sendo Ts4 longa e curvada, 

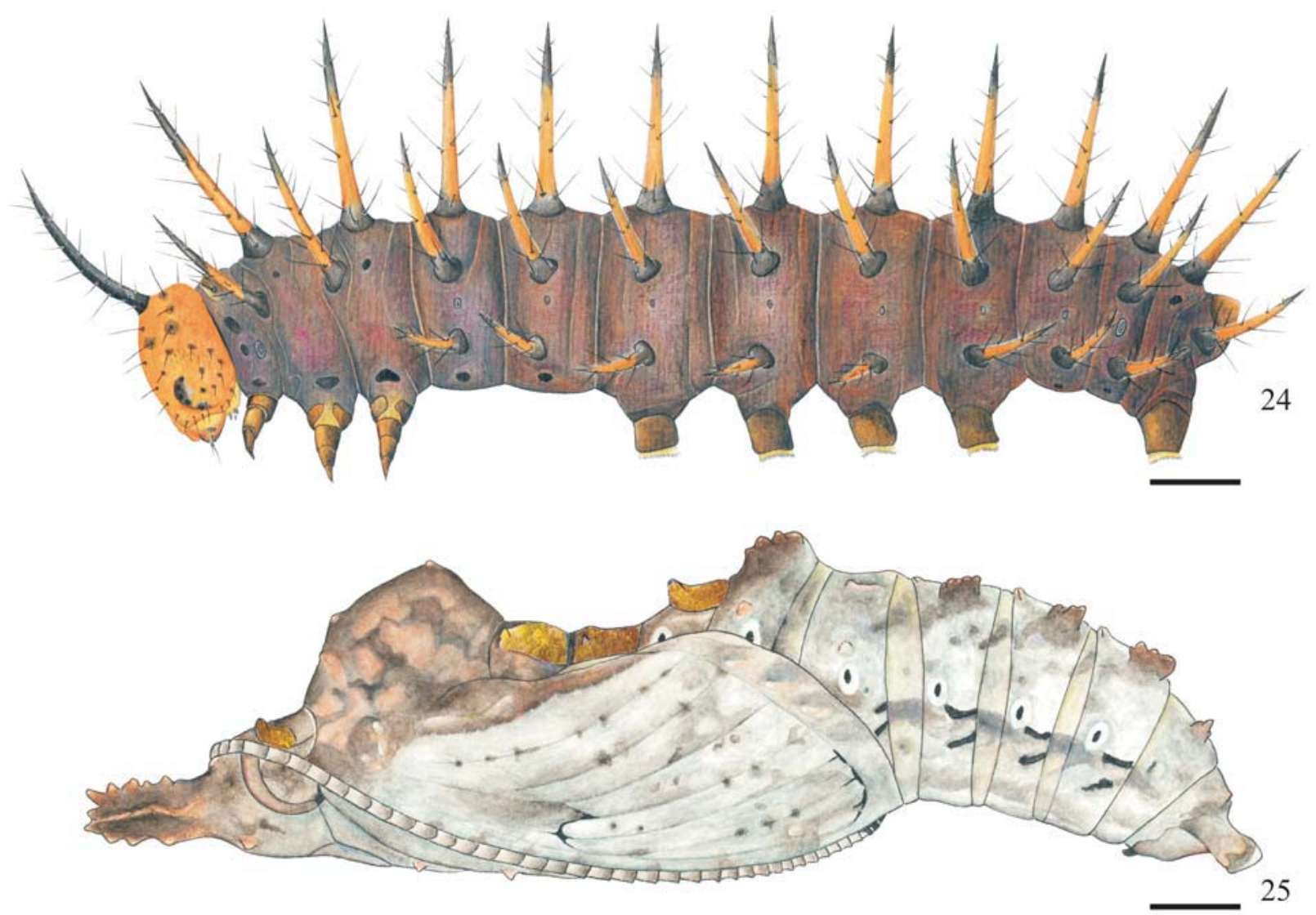

Figs. 24-25. Padrão de coloração de Dryadula phaetusa (Linnaeus, 1758). 24, larva de quinto ínstar; 25, pupa. Barras = 2,5 mm.

semelhante à garra tarsal.

Meso- e metatórax: cerdas D1 e D2 em pináculas separadas, sendo D1 maior e dorsalmente posicionada em relação a D2. Cerdas SD1 e SD2 na mesma pinácula, com SD1 maior e mais dorsal que SD2. Grupo lateral representado apenas por L1. Grupo subventral formado apenas pela cerda SV1, semelhante ao descrito para diversos Heliconiini (Antunes et al. 2002; Kaminski et al. 2002; Paim et al. 2004; Silva et al. 2006), mas diferindo do descrito para Dione, que possui também a cerda SV2 (Tavares et al. 2002; Kaminski et al. 2008).

Primeiro e segundo segmentos abdominais: grupo dorsal como nos segmentos torácicos, porém em pináculas diferentes. Grupo subdorsal representado apenas por SD1. Grupo lateral formado pelas cerdas L1 e L2 em pináculas separadas, sendo L2 situada ântero-ventralmente em relação a L1. As pináculas das cerdas laterais do abdômen são semicirculares, como já havia sido detectada por Penz (1999). Grupo subventral constituído por cerdas curtas e finas, sendo SV 1 no $1^{\circ}$ e $2^{\circ}$ segmentos e SV2 apenas no $2^{\circ}$ segmento abdominal.

Terceiro ao oitavo segmento abdominal: cerdas dorsais, subdorsais e laterais semelhantes ao encontrado nos segmentos abdominais anteriores; cerdas subventrais SV1 e SV2, curtas e finas, inseridas nas seções cilíndricas dos larvópodos do $3^{\circ}$ ao $6^{\circ}$ segmentos abdominais. No $7^{\circ}$ e $8^{\circ}$ segmentos abdominais, apenas SV1 está presente. Nono segmento abdominal: cerdas dorsais semelhantes aos demais segmentos abdominais; cerda subdorsal SD1 menor que a do segmento anterior e mais ventralmente localizada; grupo lateral com L1; subventral com SV1 formados por cerdas pequenas e afiladas, ambas em pináculas circulares. Décimo segmento abdominal: placa anal com cerdas dos grupos dorsal, D1 e D2; e do grupo subdorsal, SD1 e SD2, sendo D1 a mais longa; cerdas do grupo paraproctal, PP1 (sensu Stehr 1987), e do grupo subparaproctal, SP1 (sensu Kitching 1984), esta última ventral em relação a PP1, ambas curtas e afiladas. Seção cilíndrica do larvópodo com as cerdas PL1, PL2, PL3, PL4 e PL5, esta última na posição mais ventral, não visível em vista lateral; a quetotaxia deste segmento é semelhante à descrita para Danainae (Kitching 1984), bem como para os outros Heliconiini (Antunes et al. 2002; Kaminski et al.2002; Tavares et al. 2002; Paim et al. 2004; Silva et al. 2006).

Tegumento com microtríquias cônicas (Fig. 12), ausentes dorso-lateralmente e abundantes ventralmente e próximo aos larvópodos. Espiráculos arredondados e com o peritrema elevado (Fig. 13), presentes no protórax e do primeiro ao oitavo segmento abdominal, sendo o protorácico e o último maiores que os demais. Os larvópodos apresentam na sua região plantar, ganchos unisseriais e uniordinais, dispostos circularmente (Fig. 14).

O padrão de coloração da larva de D. phaetusa (Fig. 24) é 


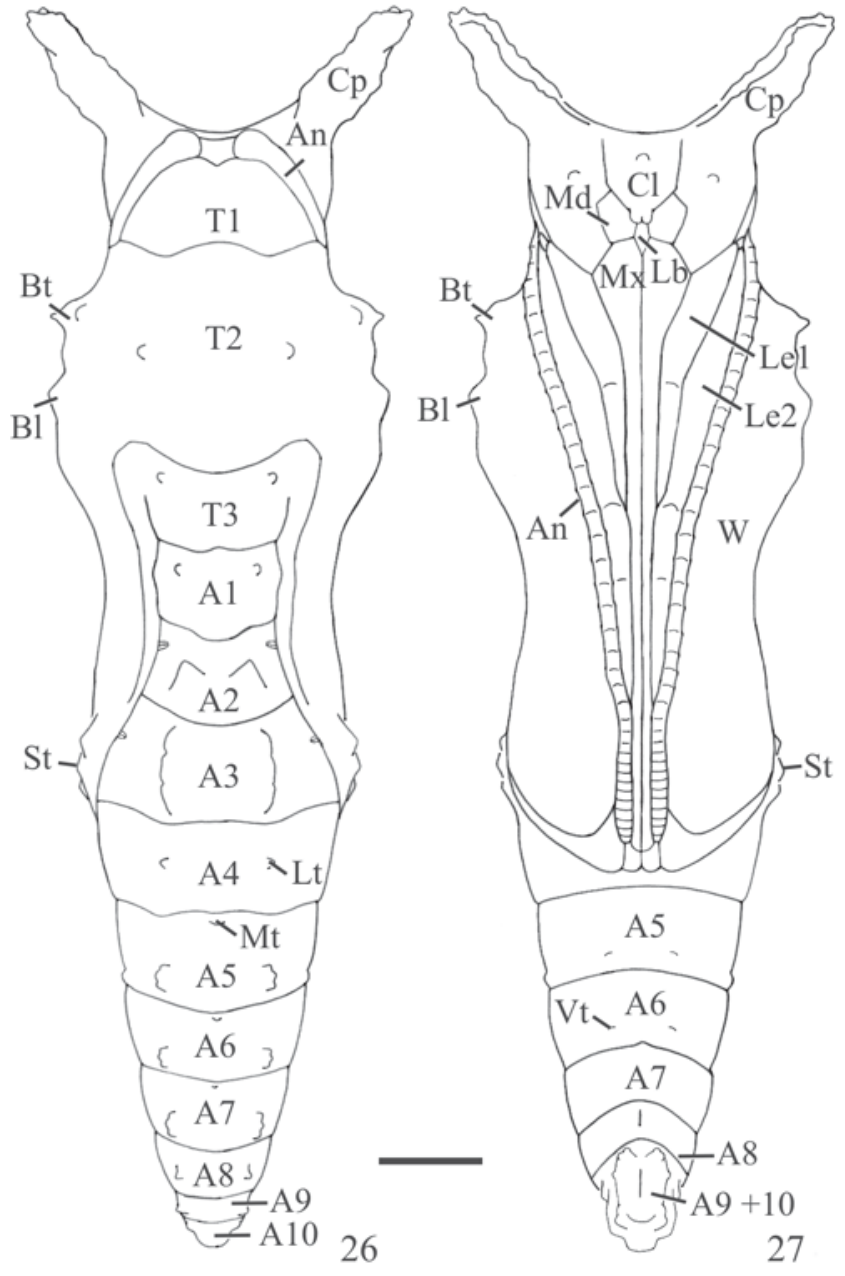

Figs. 26-27. Representação esquemática da pupa de Dryadula phaetusa (Linnaeus, 1758). 26, vista dorsal; 27, vista ventral. A, segmento abdominal; An, antena; Bl, crista longitudinal; Bt, tubérculo basilar; $\mathrm{Cl}$, clípeo; $\mathrm{Cp}$, projeção cefálica; Lb, labro; Le, perna; Lt, tubérculo láterodorsal; Md, mandíbula; Mt, tubérculo médio-dorsal; Mx, maxila; St, tubérculo submarginal; T, segmento torácico; Vt, tubérculo ventral; W, asa. Barra $=2,5 \mathrm{~mm}$

um dos mais uniformes dentre os Heliconiini, sem a presença de manchas ou faixas comuns a outras espécies. A partir do segundo ínstar, a cápsula cefálica se torna alaranjada com algumas manchas escuras na base das cerdas cefálicas e próximas aos estematas. Corpo apresenta coloração escura e aspecto aveludado, variando do cinza-alaranjado ao chumboescuro. Verrucas e base dos escolos preto-reluzentes. Pernas e seção cilíndrica dos larvópodos variando do preto ao cinzaalaranjado. Escolos cefálicos pretos, demais escolos totalmente pretos ou com uma porção mediana alaranjada.

A principal mudança morfológica que ocorre a partir do segundo ínstar é o surgimento dos escolos e verrucas em posição similar às cerdas primárias (Fig. 8). O padrão de distribuição é constante dentre os ínstares, seguindo o descrito por Beebe et al. (1960) (Fig. 8). Os escolos estão distribuídos em cinco grupos: cefálico (Sc), dorsal (So), supra-espiracular $(\mathrm{Sp})$, subespiracular $(\mathrm{Sl})$ e anal $(\mathrm{Sn})$. Os escolos são estruturas alongadas, que apresentam diversas cerdas inseridas, distinguindo-se dois tipos (Fig. 17): 1) maiores e afiladas, e 2) menores com ápice distinto, freqüentemente dilatado (Fig. 19). A porção distal dos escolos é afilada, sendo constituída por uma cerda com a base tenuemente delimitada (Fig. 18), este padrão tem sido descrito para outras larvas de Heliconiini (Penz 1999; Antunes et al. 2002; Kaminski et al. 2002; Paim et al. 2004; Silva et al. 2006).

Cápsula cefálica com um par de escolos bem desenvolvidos e curvados. Protórax sem escolos (Fig. 8), apresentando na placa protorácica três pares de cerdas distalmente afiladas (Fig. 16); verrucas localizadas em posição semelhante às pináculas do primeiro ínstar. Meso- e metatórax com pares de escolos dorsais e supra-espiraculares deslocados anteriormente (= lateral, sensu Beebe et al. $1960,=$ Sst, sensu Müller 1886), e com verrucas subdorsais e subventrais. O deslocamento destes escolos tem sido considerado uma sinapomorfia para Heliconiinae (Müller 1886; Harvey 1991).

Primeiro e segundo segmentos abdominais: pares de escolos dorsais, supra-espiraculares e subespiraculares, com verrucas subventrais. Terceiro ao sexto segmento abdominal: pares de escolos dorsais, supra-espiraculares e subespiracular, sem verrucas. Sétimo e oitavo segmentos abdominais: similares aos anteriores, com a adição de uma verruca subventral. Nono segmento abdominal: par de escolos dorsais, com verruca dorsal e subventral. Décimo segmento abdominal com um par de escolos anais e placa anal.

Similar ao primeiro ínstar, os estematas apresentam suave facetamento em "y" e um sulco margeando anteriormente a região estematal, porém com o sulco e a margem dos estematas bem marcados (Fig.15). Espiráculos elípticos, sem peritrema elevado (Fig. 20). O tegumento do quinto ínstar larval é coberto por microtríquias e cerdas secundárias esparsas. Microtríquias de dois tipos: 1) cônicas e estriadas, distalmente afiladas (Fig. 21) ou espatuladas, junto aos larvópodos; e 2) curtas e de formato achatado, com carenas proeminentes desde a base, nas localizadas próximo aos espiráculos (Fig. 22). As cerdas secundárias são curtas, semelhantes as de Agraulis vanillae maculosa (Stichel, [1908]), D. iulia alcionea, D. juno juno e $H$. erato phillys, mas diferindo do observado em Dione moneta moneta Hübner, [1825] e Dione glycera (Felder \& Felder, 1861), que possuem cerdas relativamente mais longas (Penz 1999; Kaminski et al. 2008). Ganchos da planta dos larvópodos com padrão unisserial e triordinal (Fig. 23).

Identificação dos ínstares. As larvas de D. phaetusa não apresentam variações de coloração que possibilitem uma identificação segura dos diferentes ínstares. A partir dos dados correspondentes à tendência central e de dispersão, para a largura da cápsula cefálica, dentre os ínstares, obteve-se a seguinte equação exponencial de crescimento: $\mathrm{y}=0,462$ $\mathrm{e}^{\wedge} 0,412 \mathrm{x} ; \mathrm{n}=100 ; \mathrm{r}=0,995 ; \mathrm{p}=0,0001$. A razão média de crescimento dentre os ínstares foi de 1,51, próximo ao encontrado para outros heliconíneos (Antunes et al. 2002; Kaminski et al. 2002; Tavares et al. 2002; Paim et al. 2004; Silva et al. 2006; Kaminski et al. 2008). Estas medidas de 

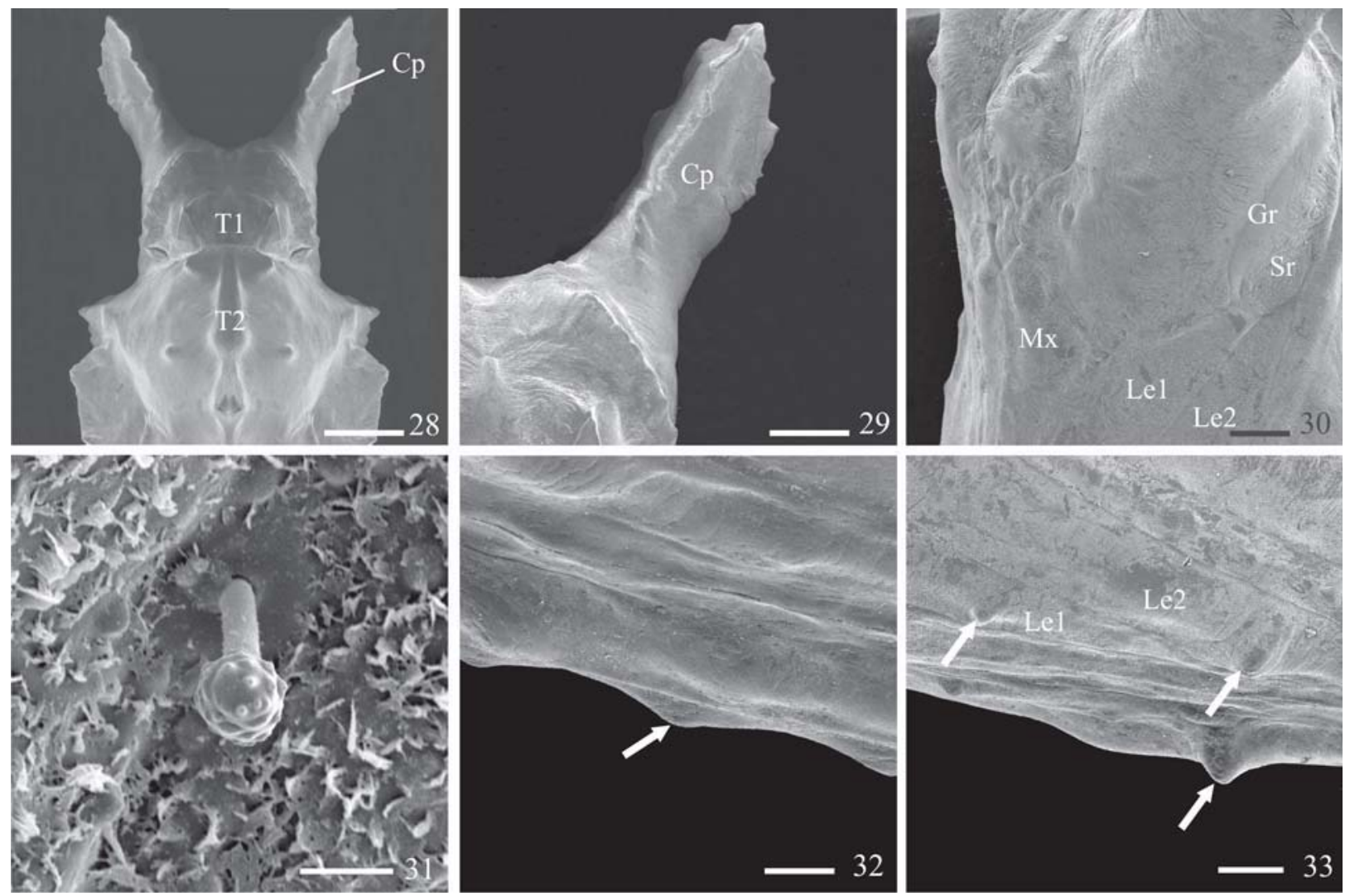

Figs. 28-33. Pupa de Dryadula phaetusa (Linnaeus, 1758). 28, cabeça e segmentos torácicos em vista dorsal; 29, projeção cefálica; 30, cabeça em vista látero-ventral; 31, cerda da região esculturada do olho; 32, tubérculos da porção mediana da antena (seta); 33, tubérculos das pernas protorácica e mesotorácica (setas). Cp, projeção cefálica; Gr, faixa de aspecto reluzente do olho; Le, perna; Mx, porção proximal da maxila; Sr, porção esculturada do olho; T, segmento torácico. Barras $=2$ e $1 \mathrm{~mm}, 500,10,500$ e $400 \mu \mathrm{m}$, respectivamente.

crescimento seguem a regra de Brooks-Dyar (Daly 1985), onde os valores se ajustam a uma curva de crescimento exponencial, sugerindo um crescimento segundo progressão geométrica. Não foi observada sobreposição dos intervalos de crescimento entre os ínstares, o que permite a identificação acurada dos mesmos através da largura da cápsula cefálica (Tab. I).

Pupa. Apresenta padrão de coloração constituído por uma mistura de tonalidades de marrom e cinza (Fig. 25). Dorsalmente, estão presentes quatro pares de manchas douradas: no protórax, metatórax, primeiro e segundo segmentos abdominais. O padrão de coloração é relativamente homogêneo, com pequenas variações de intensidade entre indivíduos.

A semelhança de outros Heliconiini, a morfologia segue o padrão geral proposto por Mosher (1916) para as pupas de Nymphalidae, sem sutura epicranial e com as pernas pro- e mesotorácicas tocando a margem posterior da região ocular (Figs. 26, 27, 30). As peças bucais se apresentam fundidas em vista ventral (Fig. 27). O labro (Lb) encontra-se em posição central, margeado lateralmente pelas mandíbulas $(\mathrm{Md})$ e anteriormente pelo clípeo (Cl). As maxilas (Mx, Fig. 30) estão posicionadas posteriormente ao labro, e são tão longas quanto as pernas. As antenas (An, Figs. 26-27, 32) se originam dorsalmente e se estendem ventralmente ao longo das asas.
Artículos antenais com pequenos tubérculos (Fig. 32). Região ocular (Figs. 25, 30) constituída por duas áreas: uma de aspecto lisa ( $\mathrm{Gr}$ ), e outra áspera com cerdas curtas ( $\mathrm{Sr}$ ), semelhante ao descrito para outros heliconíneos (Antunes et al. 2002; Tavares et al. 2002; Kaminski et al. 2002; Paim et al. 2004; Silva et al. 2006; Kaminski et al. 2008). A porção terminal destas cerdas é dilatada com pequenas pontas (Fig. 31).

Pupa pertencente ao grupo "A" de Beebe et al. (1960), constituído por espécies com tubérculos curtos sem espinhos ou flanges. No entanto, como já havia sido notado por Beebe et al. (1960), difere das demais espécies deste grupo por apresentar projeções cefálicas longas (Cp, Figs. 26-29). A presença destas projeções longas confere à pupa de $D$. phaetusa um aspecto alongado (Fig. 25) que contrasta com as pupas de outros Heliconiini próximos filogeneticamente (Penz 1999), como as de Dryas e Philaethria, que possuem aspecto mais atarracado tipo "bird dropping" (Beebe et al., 1960; Paim et al. 2004).

Pernas visíveis em vista ventral, inclusive a parte da perna metatorácica, cuja porção mais distal surge posterior às antenas; tal característica já havia sido detectada por Penz (1999). Pernas pro- e mesotorácicas com pequenos tubérculos (Figs. 27, 33). Asa com tubérculo basilar (Bt) seguido de uma crista longitudinal (Bl) na porção proximal (Figs. 26-27, 34). Próximo à margem posterior da asa, encontram-se três pares de tubérculos submarginais (St, Figs. 26-27). Mesotórax (Fig. 

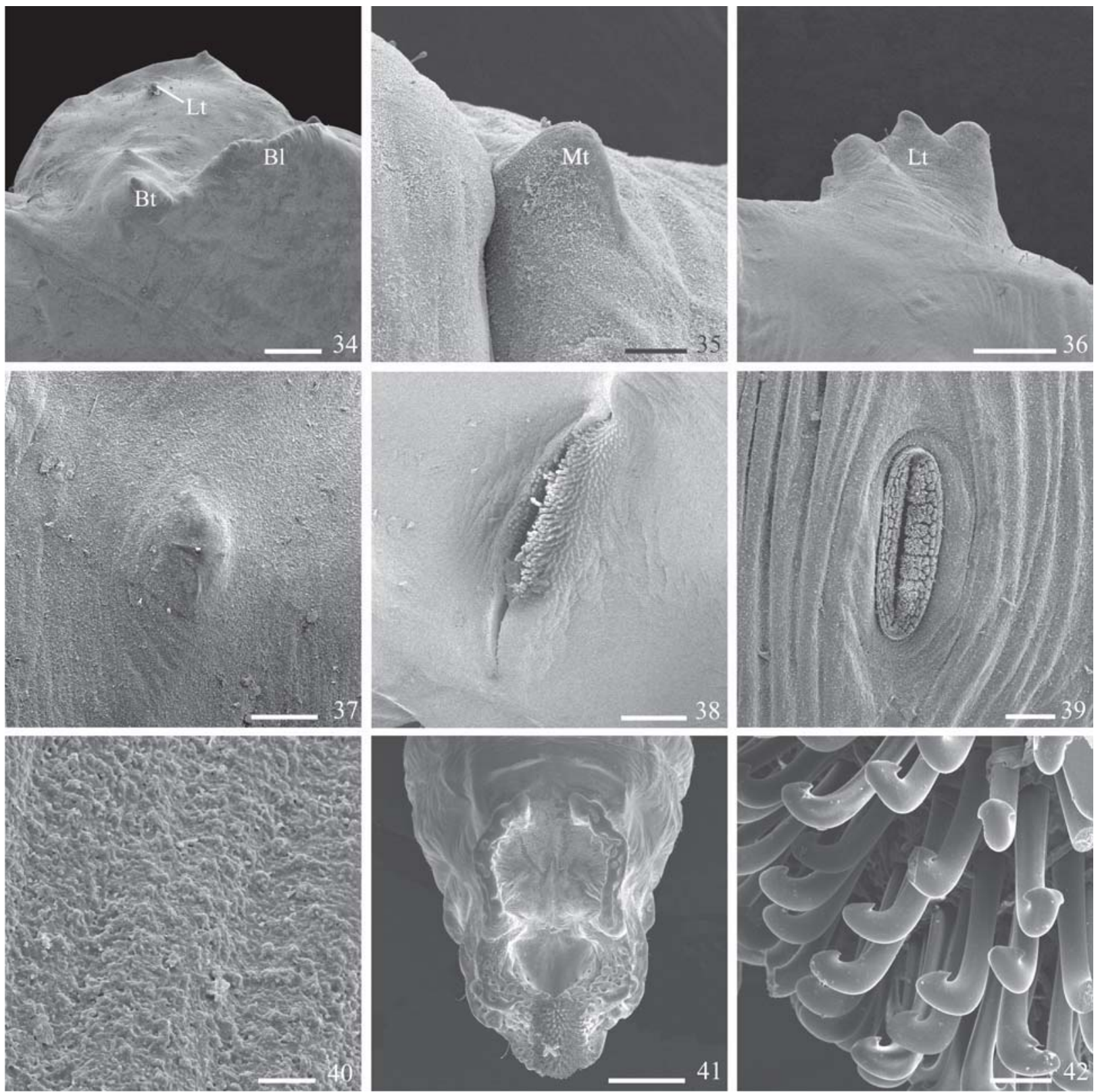

Figs. 34-42. Pupa de Dryadula phaetusa (Linnaeus, 1758). 34, mesotórax em vista látero-ventral; 35, tubérculo médio-dorsal em vista láterodorsal; 36, tubérculo látero-dorsal em vista látero-dorsal; 37, tubérculo supra-espiracular; 38 , espiráculo mesotorácico; 39, espiráculo do $5^{\circ}$ segmento abdominal; 40, detalhe da ornamentação do tegumento; 41, cremáster em vista ventral; 42, ganchos do cremáster. Bl, crista longitudinal; Bt, tubérculo basilar; Lt, tubérculo látero-dorsal; Mt, tubérculo médio-dorsal. Barras $=1 \mathrm{~mm}, 100,500,200,200,100,20,1000,50 \mu \mathrm{m}$, respectivamente.

34) pouco projetado dorsalmente, sem a presença da crista médio-dorsal, formando uma quilha, contrário ao que ocorre em Dione (Penz 1999; Tavares et al. 1999; Kaminski et al. 2008).

Principais tubérculos estão distribuídos em quatro grupos: médio-dorsal (Mt), lateral (Lt), supra-espiracular (Se) e ventral (Vt) (Figs. 26-27). Os médio-dorsais (Fig. 35) são pouco pronunciados e estão presentes na borda anterior do $5^{\circ}$ ao $7^{\circ}$ segmentos abdominais. O grupo lateral é encontrado do protórax ao $8^{\circ}$ segmento abdominal (Figs. 25-26, 36), sendo o tubérculo do $3^{\circ}$ segmento abdominal o mais proeminente. Os tubérculos supra-espiraculares (Fig. 37) são curtos e estão localizados lateralmente no $3^{\circ} \mathrm{e} 4^{\circ}$ segmentos abdominais. $\mathrm{O}$ grupo ventral (Fig. 27) é encontrado no $5^{\circ}$ e $6^{\circ}$ segmentos abdominais.

Espiráculo mesotorácico (Fig. 38), com formato de fenda sem tubérculo associado e com aparelho filtrador simples. Os demais espiráculos (Fig. 39) são elípticos com aparelho filtrador ramificado. Tegumento coberto por pequenas ornamentações (Fig. 40) que conferem um aspecto áspero para a pupa, à semelhança do descrito para D. iulia alcionea (Paim et al. 2004). Da mesma forma, o cremáster (Fig. 41) apresenta formato sub-quadrangular semelhante ao observado em Dryas, mas 
sem o truncamento posterior observado em Agraulis e Dione (Tavares et al. 2002; Silva et al. 2006; Kaminski et al. 2008). Os ganchos do cremáster (Fig. 42) são recurvados e subdivididos em duas pontas.

Agradecimentos. Os autores agradecem a Vidica Bianch pelo apoio logístico das coletas em Eldorado do Sul. Ao Centro de Microscopia Eletrônica da UFRGS e Centro de Microscopia Eletrônica e Microanálise da ULBRA, em especial a Moema Queiroz Vieira, Leandro Baum, Carlos Barboza dos Santos e Leandro Gianluppi pelo auxilio na preparação das amostras e realização das micrografias. Aos colegas do Laboratório de Morfologia e Comportamento de Insetos da UFRGS, pelas críticas e sugestões ao longo do trabalho. Ao CNPq, pelas Bolsas de Iniciação Científica concedidas a L. A. Kaminski (Proc. $\left.n^{\circ} 106325 / 2001-9\right)$ e D. S. da Silva (Proc. ${ }^{\circ} 108194 / 2003-5$ ). Este trabalho foi parcialmente financiado pelo CNPq (Proc. $\mathrm{n}^{\circ}$ 478787/2001-4).

\section{REFERÊNCIAS}

Antunes, F. A.; A. O. Menezes Jr.; M. Tavares \& G. R. P. Moreira. 2002. Morfologia externa dos estágios imaturos de heliconíneos neotropicais: I. Eueides isabella dianasa (Hübner, 1806). Revista Brasileira de Entomologia 46: 601-610.

Araújo, A. M. \& V. L. S. Valente. 1981. Observações sobre alguns lepidópteros e drosofilídeos do Parque Estadual do Turvo. Ciência e Cultura 33: 1485-1490.

Beebe, W.; J. Crane \& H. Fleming. 1960. A comparison of eggs, larvae and pupae in fourteen species of heliconiine butterflies from Trinidad, W. I. Zoologica 45: 111-154.

Benson, W. W.; K. S. Brown Jr. \& L. E. Gilbert. 1975. Coevolution of plants and herbivores: passion vine butterflies. Evolution 32: 659680 .

Brown, K. S., Jr. 1981. The biology of Heliconius and related genera. Annual Review of Entomology 26: 427-456.

Brown, K. S., Jr. 1992. Borboletas da Serra do Japi: diversidade, hábitats, recursos alimentares e variação temporal, p. 142-187. In: L. P. C. Morellato (ed.). História Natural da Serra do Japí: Ecologia e preservação de uma área florestal no sudeste do Brasil. Campinas, UNICAMP/FAPESP, $322 \mathrm{p}$

Brown, K. S., Jr. \& O. H. H. Mielke. 1972. The heliconians of Brazil (Lepidoptera: Nymphalidae). Part II. Introduction and general comments, with a supplementary revision of the tribe. Zoologica 57: $1-40$.

Daly, H. V. 1985. Insect morphometrics. Annual Review of Entomology 30: 415-438.

Dell'Erba, R.; L. A. Kaminski \& G. R. P. Moreira. 2005. O estágio de ovo dos Heliconiini (Lepidoptera: Nymphalidae) do Rio Grande do Sul, Brasil. Iheringia, Série Zoologia 95: 29-46.

DeVries, P. J. 1987. The butterflies of Costa Rica and their natural history. Papilionidae, Pieridae, Nymphalidae. Princeton, Princeton University, 327 p.

Downey, J. C. \& A. C. Allyn Jr. 1981. Chorionic sculpturing in eggs of Lycaenidae. Part I. Bulletin of the Allyn Museum 61: 1-29.

Duarte, M.; R. K. Robbins \& O. H. H. Mielke. 2005. Immature stages of Calycopis caulonia (Hewitson, 1877) (Lepidoptera, Lycaenidae, Theclinae, Eumaeini), with notes on rearing detritivorous hairstreaks on artificial diet. Zootaxa 1063: 1-31.

Emsley, M. G. 1963. A morphological study of imagine Heliconiinae (Lep.: Nymphalidae) with a consideration of the group. Zoologica 48: $85-130$.

Fleming, H. 1960. The first instar larvae of the Heliconiinae (Butterflies) of Trinidad, W. I. Zoologica 45: 91-110.

Freitas, A. V. L. \& K. S. Brown Jr. 2004. Phylogeny of the Nymphalidae
(Lepidoptera). Systematic Biology 53: 363-383.

Freitas, A. V. L. \& P. S. Oliveira. 1996. Ants as selective agents on herbivore biology: effects on the behaviour of a nonmyrmecophilous butterfly. Journal of Animal Ecology 65: 205210 .

Harvey, D. J. 1991. Higher classification of the Nymphalidae. Appendix B, p. 255-273. In: H. F. Nijhout (ed.). The development and evolution of butterfly wing patterns. Washington, Smithsonian Institution, xvi $+297 \mathrm{p}$.

Kaminski, L. A.; M. Tavares; V. G. Ferro \& G. R. P. Moreira. 2002. Morfologia externa dos estágios imaturos de heliconíneos neotropicais: III. Heliconius erato phyllis (Fabricius) (Lepidoptera, Nymphalidae, Heliconiinae). Revista Brasileira de Zoologia 19: 977-993.

Kaminski, L. A.; R. Dell'Erba \& G. R. P. Moreira. 2008. Morfologia externa dos estágios imaturos de heliconíneos neotropicais: VI. Dione moneta moneta Hübner (Lepidoptera, Nymphalidae, Heliconiinae). Revista Brasileira de Entomologia 52: 13-23.

Kitching, I. J. 1984. The use of larval chaetotaxy in butterfly systematics, with special reference to the Danaini (Lepidoptera: Nymphalidae). Systematic Entomology 9: 49-61.

Kitching, I. J. 1985. Early stages and the classification of the milkweed butterflies (Lepidoptera: Danainae). Zoological Journal of the Linnean Society 85: 1-97.

Lamas, G. 2004. Checklist: Part 4A. Hesperioidea - Papilionoidea. In: J. B. Heppner (ed.). Atlas of Neotropical Lepidoptera. Volume 5A. Gainesville, Association for Tropical Lepidoptera, Scientific Publishers, xxxiv $+428 \mathrm{p}$.

Miller, J. S. 1991. Cladistics and classification of the Notodontidae (Lepidoptera: Noctuoidea) based on larval and adult morphology. Bulletin of the American Museum of Natural History 204: $1-230$.

Mosher, E. 1916. A classification of the Lepidoptera based on characters of the pupa. Bulletin of the Illinois State Laboratory of Natural History 12: 1-165.

Motta, P. C. 2003. Phylogenetic relationships of Ithomiinae based on first-instar larvae, p. 409-429. In: C. L. Boggs; W. B. Watt \& P. R. Ehrlich (eds.). Butterflies: ecology and evolution taking flight. Chicago, University of Chicago, 739 p.

Müller, W. 1886. Südamerikanische Nymphalidenraupen. Versuch eines naturlichen Systems der Nymphaliden. Zoologischen Jahrbüchern 1: 417-678.

Paim, A. C.; L. A. Kaminski \& G. R. P. Moreira. 2004. Morfologia externa dos estágios imaturos de heliconíneos neotropicais: IV. Dryas iulia alcionea (Lepidoptera, Nymphalidae, Heliconiinae). Iheringia, Série Zoologia 94: 25-35.

Penz, C. M. 1999. Higher level phylogeny for the passion-vine butterflies (Lepidoptera: Heliconiinae) based on early stage and adult morphology. Zoological Journal of the Linnean Society 127: 277-344.

Peterson, A. 1962. Larvae of insects. An introduction to Neartic species. Part I Lepidoptera and plant infesting Hymenoptera. Ann Arbor, Edwards Brothers Inc., 315 p.

Silva, D. S. da; R. Dell'Erba; L. A. Kaminski \& G. R. P. Moreira. 2006. Morfologia externa dos estágios imaturos de heliconíneos neotropicais: V. Agraulis vanillae maculosa (Lepidoptera, Nymphalidae, Heliconiinae). Iheringia, Série Zoologia 96: 219228 .

Snedecor, G. W. \& W. G. Cochran. 1980. Statistical methods. Ames, Iowa State University, $507 \mathrm{p}$.

Stehr, F. W. 1987. Order Lepidoptera, p. 288-305. In: F. W. Stehr, (ed.). Immature insects. Vol. I. Dubuque, Kendall/ Hunt Publishing Company, $975 \mathrm{p}$

Tavares, M., L. A. Kaminski \& G. R. P. Moreira. 2002. Morfologia externa dos estágios imaturos de heliconíneos neotropicais: II. Dione juno juno (Cramer) (Lepidoptera: Nymphalidae: Heliconiinae). Revista Brasileira de Zoologia 19: 961-976. 